\title{
Atuação do enfermeiro na prescrição de contraceptivos hormonais na rede de atenção primária em saúde
}

\author{
Performance of nurses in prescribing hormonal contraceptives in the primary health care network
} Acción de enfermeros en la prescripción de anticonceptivos hormonales en la red de atención primaria de salud

\section{Jamille Gregório Dombrowski', Jéssika Abrantes Pontes", Walédya Araújo Lopes de Melo e Assis ${ }^{\text {III }}$}

' Universidade de São Paulo, Instituto de Ciências Biomédicas, Departamento de Parasitologia, Programa de Biologia da Relação Patógeno-Hospedeiro (Doutoranda). São Paulo-SP, Brasil.

"Centro de Educação Técnica e Especializada do Acre, Curso de Especialização Técnica de Enfermagem no Trabalho. Rio Branco-AC. Brasil.

I"' Universidade Federal do Acre, Departamento de Ciências da Saúde e Educação Física, Curso de Medicina. Rio Branco-AC, Brasil.

\section{Submissão: 21-11-2012 Aprovação: 01-12-2013}

\section{RESUMO}

O estudo teve como objetivo conhecer e analisar a atuação do enfermeiro na prescrição dos contraceptivos hormonais reversíveis na Rede de Atenção Primária a Saúde. Trata-se de um estudo transversal e descritivo, tendo como sujeitos 64 enfermeiros lotados nas unidades assistenciais no período de setembro a novembro de 2010 no município de Rio Branco-Acre. Os dados foram coletados mediante entrevistas estruturadas utilizando um questionário. Os resultados evidenciaram que $96,9 \%$ dos enfermeiros prescrevem os métodos anticoncepcionais e que 90,6\% tem conhecimento da legislação que rege a prescrição de enfermagem. Foi observado que a escolha do método contraceptivo pelos enfermeiros baseia-se na escolha da cliente e anamnese (36\% e 34\% respectivamente), e que 90\% sempre orientavam quanto às vantagens e desvantagens de cada método. Assim, para escolher um método contraceptivo de forma livre e informada, os métodos devem estar disponíveis e devem ser dispensados por profissionais capacitados, após orientação correta e completa.

Descritores: Anticoncepção; Papel do Profissional de Enfermagem; Atenção Primária à Saúde.

\section{ABSTRACT}

The aim of this study was to evaluate the performance of nurses in the prescription of hormonal reversible contraceptives in Primary Care. A descriptive and transversal study was conducted, with 64 nurses at healthcare units as subjects, in the period of September-November 2010 in the county of Rio Branco-Acre. Data were collected through structured interviews using a questionnaire. The results showed that $96.9 \%$ of nurses are prescribing hormonal contraceptives and that the majority (90.6\%) is aware of the government laws about nursing prescription. We also observed that the contraceptive was chosen based on client's decision and anamnesis ( $36 \%$ and $34 \%$ respectively), and that $90 \%$ of the nurses always give orientation about advantages and disadvantages of each contraceptive method. Thus, for choosing a contraceptive method based on given information and free will, the methods must be available and dispensed by trained professionals, after correct and complete orientation.

Key words: Contraception; Nurse's Role, Primary Health Care.

\section{RESUMEN}

El estudio tuvo como objetivo identificar y analizar el desempeño de los enfermeros en la prescripción de anticonceptivos hormonales reversibles en la Red de Atención Primaria. Se trata de un estudio descriptivo y transversal, que tiene como sujetos 64 enfermeros inscritos en las unidades de cuidado de salud en el período de septiembre a noviembre de 2010 en la ciudad de Rio Branco, Acre. Los datos fueron recolectados a través de entrevistas con un cuestionario estructurado. Los resultados mostraron que $96,9 \%$ de las enfermeras recetan los anticonceptivos y que $90,6 \%$ tiene conocimiento de las leyes que regulan la prescripción de enfermería. Se observó que la elección del método anticonceptivo por las enfermeras es basada en la decisión del cliente y la anamnesis (36\% y 34\% respectivamente) y que $90 \%$ siempre aconsejaban a respecto de las ventajas y desventajas de cada método. Así que para elegir un método anticonceptivo de manera libre e informada, todos los métodos deben estar disponible y ser dispensados por profesionales capacitados, después de la orientación correcta y completa.

Palabras clave: Anticoncepción; Rol de la Enfermera; Atención Primaria de Salud. 


\section{INTRODUÇÃO}

A assistência ao planejamento familiar no Brasil é oferecida pela rede de atenção primária à saúde como parte integrante do modelo de descentralização do Sistema único de Saúde, sendo uma das sete áreas prioritárias de intervenção na atenção primária definida na Norma Operacional da Assistência ${ }^{(1)}$.

O Ministério da Saúde, tomando por base o dispositivo da lei do planejamento familiar (Lei $n^{\circ} .9 .263 / 96$ ), determina como competência dos profissionais de saúde, assistir em concepção e contracepção, empenhando-se em informar os indivíduos sobre as opções e finalidades dos métodos disponíveis. A atuação dos profissionais de saúde neste âmbito deve, também, estar pautada no princípio da paternidade responsável e no direito de livre escolha dos indivíduos e/ou casais, obedecendo ao Artigo 226, Parágrafo 7, da Constituição da República Federativa do Brasil ${ }^{(2)}$.

$\mathrm{O}$ acesso à informação de boa qualidade e a disponibilidade de alternativas contraceptivas são aspectos fundamentais nos programas de planejamento familiar à população em geral. $\mathrm{O}$ conhecimento inadequado sobre qualquer método anticoncepcional pode ser um fator de resistência à aceitabilidade e uso do método. Do mesmo modo, o alto nível de conhecimento sobre os métodos anticoncepcionais (MAC) não determinará nenhuma mudança de comportamento se estes não estiverem acessíveis à livre escolha da população ${ }^{(3)}$.

A opção por um MAC implica tanto o número de métodos oferecidos quanto a sua variedade, em termos das características intrínsecas de cada um. Desta forma, para que as pessoas, de fato, tenham opção e possam escolher livremente, é relevante também a informação científica recebida e assimilada acerca dos MAC disponíveis. Por outro lado, na prática assistencial, tem se verificado que as mulheres quando pretendem usar um método específico, chegam aos serviços de saúde com a decisão tomada e não se mostram receptivas a uma orientação ampla e completa acerca de todas as alternativas de MAC disponíveis ${ }^{(4)}$.

Para que haja uma boa gestão do cuidado a competência profissional no campo da anticoncepção deve-se incluir conhecimentos técnicos, científicos e culturais atualizados, direcionados ao atendimento das necessidades da saúde sexual e reprodutiva dos clientes. Isso inclui habilidade para dar orientação, informar e comunicar-se adequadamente, participando da tomada de decisões quanto aos MAC e acolhendo com respeito o/a cliente ${ }^{(5)}$.

É preciso à implementação de políticas públicas de planejamento familiar que reconheçam o potencial do enfermeiro em manejar os métodos anticoncepcionais e explicitem seu amparo legal para que assuma com autonomia essa área do cuidado para a qual soma grande contribuição ${ }^{(6)}$.

Tendo em vista esses aspectos, o objetivo deste estudo foi conhecer e analisar a atuação do enfermeiro na prescrição dos contraceptivos hormonais reversíveis na Rede de Atenção Primária a Saúde, observando a legislação vigente, condições de trabalho e capacitação dos enfermeiros.

\section{METODOLOGIA}

Trata-se de um estudo transversal, realizado no período de setembro a novembro de 2010 no município de Rio Branco, capital do Estado do Acre. A área de abrangência da assistência primária à saúde na capital é de 13 (treze) distritos urbanos e 3 (três) rurais através da modalidade gestão plena da atenção primária, o que gera um percentual na cobertura dos serviços de saúde de 50,4\% da população.

A distribuição dos profissionais nesse período foi de 45 enfermeiros no Programa de Saúde da Família (PSF) e 65 enfermeiros nos Centros de Saúde, totalizando 110 profissionais (dados fornecidos pela Secretaria Municipal de Saúde de Rio Branco - SEMSA). Foram excluídos os profissionais que se encontravam licenciados, em período de férias ou que se recusaram a participar do estudo. Assim, a amostra foi composta por 64 enfermeiros que estavam lotados nas unidades assistenciais do município.

Todos os participantes assinaram o Termo de Consentimento Livre e Esclarecido e a coleta de dados foi realizada mediante entrevistas no próprio local de trabalho com cada profissional com preenchimento de questionário estruturado.

Os dados obtidos no inquérito foram processados em pacote estatístico SPSS (Statistical Package for Social Science), versão 13.0 for Windows. A partir dos resultados obtidos foi calculada a prevalência de prescrição por parte dos profissionais entrevistados e o percentual de enfermeiros que tem conhecimento sobre a legislação que rege a prescrição da enfermagem. Em complemento, foi analisada a atuação dos enfermeiros da atenção primária na orientação quanto ao uso de anticoncepcionais, com ênfase na existência ou não de orientação e/ou notificação de reações adversas.

Conforme prerrogativas da Resolução no 196/96 do Conselho Nacional de Saúde, o estudo foi aprovado pelo Comitê de Ética em Pesquisa da Universidade Federal do Acre sob protocolo $n^{\circ} 23107.013171 / 2010-96$.

\section{RESULTADOS}

Segundo a Lei do Exercício Profissional do Enfermeiro 7.498/1986 e a Resolução COFEN n 271/2001, o enfermeiro pode prescrever os anticoncepcionais hormonais orais; todavia, os hormonais injetáveis, quando utilizados pela primeira vez, seriam transcrição da prescrição, já que a cliente deve passar necessariamente por uma consulta médica (Fluxograma 1).

Fluxograma 1 - Possibilidades de prescrição do enfermeiro.

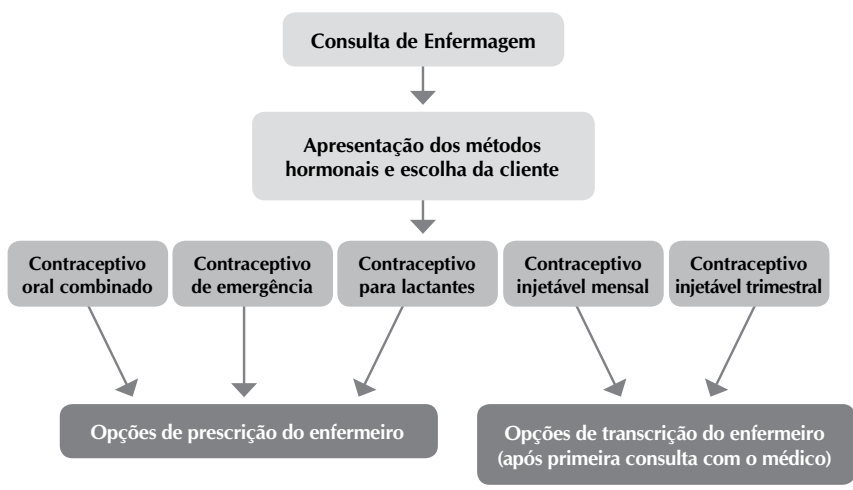

Fonte: Lei do Exercício Profissional do Enfermeiro 7.498/1986 e Resolução COFEN no 271/2001. 
Os enfermeiros lotados nas unidades de atenção primária à saúde do município de Rio Branco foram questionados quanto à prescrição de anticoncepcionais. A maioria $(96,9 \%)$ respondeu que prescreviam anticoncepcionais e somente $3,1 \%$ relataram que não realizavam a prescrição.

Dentre os profissionais que realizavam prescrição dos MAC, apenas 15 enfermeiros afirmaram que prescreviam somente os anticoncepcionais hormonais orais e que os injetáveis eram transcritos após avaliação médica. Um achado interessante foi o fato de todos os enfermeiros relatarem a escassa participação dos profissionais médicos nas atividades de planejamento familiar ou a inexistência desse profissional nas unidades de saúde, o que dificultava o fluxo correto de atendimento.

A respeito do conhecimento da legislação que rege a prescrição de enfermagem, 90,6\% dos enfermeiros disseram que conheciam a legislação vigente e apenas 9,4\% não tinham conhecimento pleno sobre esta. Dentre as respostas positivas, podemos dividir os profissionais em dois grupos importantes: os enfermeiros que tinham total conhecimento sobre a legislação e os que disseram que sabiam da sua existência, mas não sabiam citá-la. Observou-se então a falta de capacitação dos enfermeiros quanto à disciplina de deontologia da enfermagem, seu respaldo legal.

Para a avaliação da clínica utilizada pelo enfermeiro, foi questionado em que era baseada a escolha do anticoncepcional. As mais diversas respostas foram agrupadas em quatro categorias: anamnese, idade da cliente, disponibilidade do MAC na unidade e escolha da cliente (Gráfico 1). A resposta "escolha da cliente" foi a mais utilizada para prescrição dos MAC.

\section{Gráfico I - Avaliação Clínica}

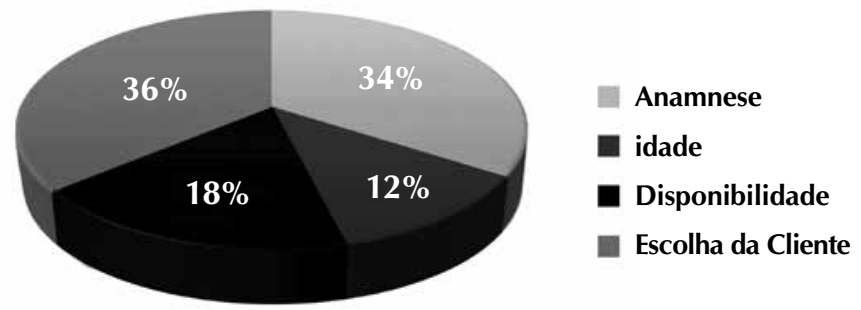

Em relação à capacitação profissional para escolha e prescrição dos MAC, 90,6\% dos enfermeiros afirmaram que estão no serviço há bastante tempo, além de terem fácil acesso aos Manuais do Ministério da Saúde e outros livros de consulta. Apenas 9,4\% responderam que não se sentiam capacitados, devido a pouca experiência e principalmente porque os métodos hormonais estão em constante atualização e desta forma precisam de um cuidado especial no seu manejo.

Quando os enfermeiros foram questionados sobre a existência de orientação durante a dispensação do anticoncepcional, e se havia notificação de reações adversas, a maioria $(90 \%)$ foi enfática afirmando que havia orientação, principalmente sobre vantagens e desvantagens do método e as possíveis reações adversas que poderiam ocorrer.
Quanto à notificação, alguns disseram que nunca notificaram nenhuma reação devido não existir um feedback entre a mulher e o enfermeiro, mesmo quando este solicita seu retorno à unidade de saúde; outros argumentaram que não existe nenhum documento específico para notificação de reações adversas para os contraceptivos; e uma pequena parcela realizava as anotações no prontuário da cliente e alterava o MAC ou encaminhava para o médico quando ocorria algum desconforto com o método.

Observando a necessidade de elaboração de rotinas e protocolos para o serviço de enfermagem da Rede de Atenção Primária a Saúde do município de Rio Branco, relacionado ao planejamento familiar, perguntou-se ao enfermeiro se o mesmo os utilizaria como balizador na escolha e prescrição dos anticoncepcionais hormonais reversíveis. Os resultados foram: 96,9\% afirmaram que utilizariam, pois teriam mais respaldo legal e segurança na escolha e prescrição dos contraceptivos, além de ser uma Sistematização da Assistência em Enfermagem; e 3,1\% disseram que não utilizariam, pois já existia a Lei $n^{\circ} 7.498 / 1986$ e não havia necessidade da criação de mais um documento.

\section{DISCUSSÃO}

A prescrição de medicamentos é uma atividade praticada pelo enfermeiro como integrante da equipe de saúde. No entanto, os limites legais para a prática desta ação são os Programas de Saúde Pública e rotinas que tenham sido aprovadas em instituições de saúde, pública ou privada ${ }^{(7)}$. Com isso, um estudo mostrou que sem conhecer os seus limites e/ ou práticas profissionais, devido ao simples fato de não deter conhecimentos necessários de sua legislação profissional, leva o próprio enfermeiro a reduzir e limitar sua autonomia profissional $^{(8)}$.

Na rede de atenção primária em Rio Branco-AC, podem-se perceber apenas três tipos de atendimento, em contraste com uma pesquisa realizada no estado do Ceará, no qual foram identificados cinco variedades de atendimento: (1) a prescrição dos MAC por enfermeiros em 48,3\% das equipes; (2) a prescrição da maioria dos MAC por enfermeiros, exceto o injetável, em 17,2\% das equipes; (3) a prescrição dos MAC por enfermeiros, à exceção do injetável e do anovulatório oral, em 13,8\% das equipes; (4) a prescrição dos MAC somente pelo médico em 13,8\% das equipes; e (5) a entrega dos MAC por enfermeiros que não realizavam a prescrição formalmente por temerem denúncia em $6,9 \%$ equipes ${ }^{(6)}$.

Nesta mesma pesquisa, foi observado que parte dos enfermeiros que prescrevia os métodos anticoncepcionais atribuiu o fato à inexistência de médico na equipe, a falta de dedicação em tempo integral a Unidade Básica de Saúde e ao pouco envolvimento destes nas ações de planejamento familiar ${ }^{(6)}$, corroborando com achados do presente estudo. $\mathrm{O}$ enfermeiro que possuía competência realizava a prescrição dos MAC, sem amparo legal, devido à solicitação das usuárias e necessidade da comunidade ${ }^{(6)}$.

Isso demonstra que a prescrição e a dispensação dos MAC continuam associadas às barreiras institucionais e 
profissionais. Percebe-se que a ausência de médico ou a alta rotatividade, além do pouco envolvimento nas atividades de planejamento familiar e cumprimento parcial de carga horária, afetavam a atividade de enfermagem quanto ao mecanismo de prescrição.

De acordo com entendimento do Conselho Internacional de Enfermeiros, para que o enfermeiro possa exercer práticas avançadas de enfermagem, inclusive a prescrição de medicamentos, deveria ter como mínimo de formação o grau de mestre em enfermagem, mestrado profissionalizante ou alguma forma de pós-graduação em práticas avançadas ou especializadas de enfermagem. Seria, pois, um profissional com conhecimento especializado e habilidade para decisões complexas, além de competência clínica para a prática de atividades expandidas, cujas características estariam conformadas pelo contexto institucional onde esteja habilitado a exercer a atividade profissional ${ }^{(9)}$.

O Ministério da Saúde, em relatório de avaliação das Equipes de Saúde da Família, revelou que mais da metade dos profissionais médicos e enfermeiros, atuantes em saúde da mulher, não foram capacitados para as ações em planejamento familiar, tratamento de afecções ginecológicas, prevenção de câncer de colo uterino e consultas pré-natal|(10).

Apesar da maioria dos enfermeiros já ter tido acesso a cursos sobre a temática em estudo, quase a totalidade afirmou alguma debilidade no desempenho de suas atividades. A capacitação é um dos meios para melhorar a competência técnica, entretanto, não é sinônimo de qualidade ${ }^{(5)}$. Com a escassez de médico nas Unidades Básicas de Saúde e uma política expansionista aliada à desorientação dos programas de educação continuada, instituiu-se portarias que regulamentam as práticas de enfermagem com intuito de suprir a necessidade do serviço, e assim aumentando ainda mais as responsabilidades do enfermeiro tornando-o cauteloso em suas atividades ${ }^{(8)}$.

Foi visto que, para a prescrição dos MAC, a maioria dos enfermeiros aceita a escolha da cliente, respeitando o Artigo 226, Parágrafo 7, da Constituição da República Federativa do Brasil, em relação ao direito de livre escolha dos indivíduos e/ou casais ${ }^{(2)}$, além de utilizarem outras clínicas como anamnese, idade da cliente e disponibilidade do MAC na unidade.

Considera-se como direito reprodutivo o

direito das pessoas de decidirem, de forma livre e responsável, se querem ou não ter filhos, quantos filhos desejam ter e em que momento de suas vidas. Direito a informações, meios, métodos e técnicas para ter ou não ter filhos. Direito de exercer a sexualidade e a reprodução livre de discriminação, imposição e violência.

Os profissionais de saúde devem oferecer subsídios para a efetivação destes direitos ${ }^{(11)}$.

Já foi demonstrado, corroborando os resultados encontrados neste trabalho, que o planejamento familiar é um conjunto de ações em que são oferecidos recursos tanto para a concepção quanto para a contracepção ${ }^{(11-12)}$. Esses recursos devem ser cientificamente aceitos e não colocar em risco a vida da pessoa, com garantia da liberdade de escolha. Nesse sentido, é importante que as mulheres recebam informações corretas e completas, compreendam os riscos e benefícios que cada método oferece, podendo, assim, encontrar alternativas quanto à aceitação e escolha do método desejado.

A ação educativa em saúde é uma das atividades inerentes à enfermagem, desempenhada em toda sua área de atuação, que deve ser desenvolvida em todos os níveis de atenção à saúde. A respeito da prática educativa, os profissionais de saúde e a equipe de enfermagem devem empenhar-se nas informações precisas aos usuários, para que tenham conhecimento sobre todas as alternativas de anticoncepção e possam participar livre e ativamente da escolha do método contraceptivo(13).

Sobre a escolha da cliente, foi observado que das mulheres que procuraram o atendimento já com o MAC pretendido em mente, optaram pelo mesmo método que pensavam em escoIher quando procuram o serviço ${ }^{(14)}$.

Segundo estudo feito com usuárias de um serviço de saúde $^{(15)}$, de quase todas as mulheres que chegaram ao ambulatório já com o MAC escolhido, poucas mudaram de método, entendendo-se que as mulheres utilizavam o conteúdo recebido na prática educativa como uma espécie de legitimador do que já tinham em mente. Esses resultados levam à reflexão sobre qual o papel das práticas educativas, já que as mulheres chegam ao serviço com pré-concepções sobre os métodos contraceptivos e acabam escolhendo o mesmo método que queriam quando chegaram para o atendimento do planejamento familiar ${ }^{(14)}$.

A falta de disponibilidade do contraceptivo nas unidades básicas de saúde não é uma realidade só do município de Rio Branco-AC. Uma pesquisa demonstrou as dificuldades de obtenção dos métodos no serviço de planejamento familiar em estudo realizado em Salvador. Os dados revelaram que $40 \%$ das entrevistadas tinham dificuldades de obtenção do anticoncepcional injetável, seguindo $20 \%$ do oral e $10 \%$ do preservativo $^{(16)}$. A opção das mulheres pela esterilização pode ser indicativa de rejeição as alternativas oferecidas pelos serviços de saúde, na busca de assegurar o controle sobre sua fecundidade.

Um amplo estudo realizado em quatro capitais brasileiras, mostrou dificuldades de comunicação com o Ministério da Saúde para solicitar recursos, informações ou revisão dos quantitativos de métodos enviados ao município. Foi relatado ainda que, o suprimento dos insumos era de responsabilidade compartilhada entre município, estado e Ministério da Saúde. No entanto, os gestores e profissionais entendiam que a responsabilidade era somente do Ministério da Saúde. Os municípios necessitam ser responsabilizados pela aquisição dos MAC, pela demora na irregularidade e insuficiência dos recursos. Foi apontado também o excesso de burocracia para compra desses insumos em alguns municípios repercutindo na falta dos métodos nas unidades de saúde ${ }^{(17)}$.

Segundo o anexo IV da Portaria $n^{\circ} 4.217$, que aprova as normas de financiamento e execução do Componente Básico da Assistência Farmacêutica, é de responsabilidade do Ministério da Saúde o financiamento, aquisição e distribuição de todos os MAC disponibilizados. Foi observado que a pequena variedade e/ou a oferta irregular dos MAC representa um 
obstáculo ao manejo das contraindicações e da livre escolha do método, uma vez que as usuárias se arriscam a receber o método que estiver disponível, como também demonstrado por outro estudo(5).

Neste trabalho, a maioria dos enfermeiros relatou que ofereciam todas as orientações necessárias sobre os MAC. Foi evidenciado em outro estudo ${ }^{(18)}$ que. dentre os enfermeiros entrevistados sobre as atividades que desenvolvem na assistência ao planejamento familiar, 51,7\% destacaram a consulta de enfermagem com seu componente de orientação individual; outros $48,3 \%$ enfatizaram realizar, além da consulta de enfermagem, trabalhos com grupos, porém reconhecendo realizarem esporadicamente. Isso mostra que uma das principais atividades do enfermeiro é a orientação aliada à educação em saúde.

Ficou evidenciada nesta pesquisa a falta da atividade dos enfermeiros no que tange à notificação de efeitos adversos. Recentemente, foi observado que os eventos adversos foram notificados por profissionais de saúde $(51 \%)$, por usuários ( $32 \%$ ) e em $17 \%$ das notificações não foi informada a origem. Dentre os profissionais de saúde, $58,8 \%$ das notificações são provenientes de farmacêuticos, $33,3 \%$ de médicos e $7,8 \%$ de enfermeiros ${ }^{(19)}$. Este estudo demonstrou que os enfermeiros são os que menos realizam notificações dos efeitos adversos.

A Organização Mundial da Saúde define o conceito de farmacovigilância como sendo a ciência relativa à detecção, avaliação, compreensão e prevenção dos efeitos adversos ou quaisquer problemas relacionados a medicamentos. A farmacovigilância visa detectar precocemente eventos adversos conhecidos ou não, monitorando também possíveis aumentos na incidência dos $\operatorname{casos}^{(19)}$.

O enfermeiro deve monitorar quaisquer alterações que os contraceptivos possam causar na saúde dos seus clientes, tendo como responsabilidade realizar as anotações cabíveis, podendo, por exemplo, aconselhar uma cliente a mudar de método contraceptivo ${ }^{(20)}$.

Este trabalho também revelou que os enfermeiros não se sentem capacitados, gerando insegurança nas ações prescritivas, apesar de existir um Protocolo do Ministério da Saúde que regulamenta o ato do Enfermeiro prescrever dentro dos programas de saúde, com atribuições específicas na Estratégia Saúde da Família normatizadas ${ }^{(9)}$.

Com isso, para atuar na assistência ao planejamento familiar nas unidades básicas, é necessário elaborar rotinas e protocolos reconhecidos e acatados pela Secretaria de Saúde do Município. A participação do gestor municipal é significante na validação dos protocolos e rotinas elaborados pelos enfermeiros, a fim de que possam exercer com autonomia suas atribuições profissionais, amparados pelos Conselhos Regionais e Federal de Enfermagem e normatizações do Ministério da Saúde em relação aos programas ${ }^{(12)}$.

Após pesquisa bibliográfica em sites de busca e nas bases de dados MEDLINE/PubMed, LILACS e SciELO, sobre o assunto em questão, verificou-se que esta necessidade presente no município de Rio Branco também é descrita em outros locais do país, sendo encontrado pouquíssimos protocolos terapêuticos para o fornecimentos de métodos contraceptivos reversíveis, localizados principalmente na região sul e sudeste.
O protocolo se torna uma instrução escrita específica para administração de determinados medicamentos em uma determinada situação clínica, que neste estudo seriam os anticoncepcionais hormonais. Essa instrução pode ser elaborada dentro de qualquer instituição, pública ou privada, e aprovada pelo dirigente local. O protocolo é amplamente usado no Reino Unido, Austrália e Nova Zelândia, além do Brasil. O uso do protocolo pode ser o caminho para que enfermeiros possam futuramente prescrever mais e de forma independente ${ }^{(8)}$.

\section{RECOMENDAÇÕES}

Para uma melhor assistência no planejamento familiar, um estudo propõe uma modalidade de capacitação com base em competências, com monitoramento periódico em serviço, no qual se avalia o nível de aprendizagem e de realização do participante e não o tempo gasto na capacitação ou o que foi assimilado pelo profissional(5).

Todos os profissionais que trabalham na área da saúde devem ser capacitados permanentemente, em virtude do surgimento de novos conceitos capazes de assegurar a aplicabilidade de novas estratégias para atender a qualidade e as necessidades na demanda do serviço. O impedimento técnico é um aspecto denunciado em vários estudos que confirmaram a necessidade de treinamento e reciclagem de médicos e enfermeiros que lidam com o planejamento familiar no Brasil( ${ }^{(6)}$.

A resolução do impasse legal quanto à prescrição dos métodos anticoncepcionais merece uma tomada de decisão urgente, pois configura barreira da comunidade aos MAC e transtorno legal e ético ao profissional ${ }^{(6)}$. É preciso, pois, uma política nacional de planejamento familiar que reconheça o potencial do enfermeiro em manejar os métodos anticoncepcionais e explicite seu amparo legal para que assuma com autonomia essa área do cuidado para a qual soma grande contribuição.

\section{CONCLUSÃO}

Para escolher um método contraceptivo de forma livre e informada, cada indivíduo precisa conhecer e ter acesso a todos os métodos contraceptivos cientificamente comprovados e disponíveis, adotando aquele que seja mais adequado às suas particularidades e condições de vida. As informações analisadas no presente estudo permitiram observar que o profissional precisa estar capacitado durante a prescrição do anticoncepcional escolhido pela cliente. Isto só se torna possível a partir da aquisição de conhecimento técnico-científico sobre as principais reações adversas, indicações e contraindicações e formas de orientação por parte do enfermeiro quanto ao uso de contraceptivos hormonais, sendo esta uma das principais atividades desse profissional.

Os protocolos clínicos são resultado de consenso técnico-científico e são formulados dentro de rigorosos parâmetros de qualidade, precisão de indicação e posologia. Eles desempenham um importante papel para a melhoria da qualidade da atenção à saúde, para a prescrição segura e eficaz, para a atualização e democratização do conhecimento e para a 
melhoria da qualidade da informação prestada. Além de promover o uso racional dos medicamentos por meio de regulamentação de indicações e esquemas terapêuticos, estabelecendo mecanismos de acompanhamento de uso e de avaliação de resultados. Portanto, a utilização de protocolos clínicos proporcionará uma melhora na qualidade da assistência prestada pelo profissional de Enfermagem durante as consultas de Planejamento Familiar.

\section{REFERÊNCIAS}

1. Ministério da Saúde. Guia Prático do Programa Saúde da Família. Brasília, DF: Ministério da Saúde; 2001.

2. Brasil. Lei $n^{\circ}$. 9.263, de 12 de janeiro de 1996. Regula o $\S 7^{\circ}$ do artigo 226 da Constituição Federal, que trata do planejamento familiar, estabelece penalidades, e dá outras providências. Diário Oficial da União 15 jan 1996; Seção 1. p. 561-70.

3. Martins LBM, Paiva LC, Osis MJD, Sousa MH, Neto AMP, Tadini V. Conhecimento sobre métodos anticoncepcionais por estudantes adolescentes. Rev Saúde Pública. 2006; 40(1):57-64.

4. Espejo X, Tsunechiro MA, Osis MJD, Duarte GA, Bahamondese L, Sousa MH. Adequação do conhecimento sobre métodos anticoncepcionais entre mulheres de Campinas, São Paulo. Rev Saúde Pública. 2003;37(5):583-590.

5. Moura ERF, Silva RM. Competência profissional e assistência em anticoncepção. Rev Saúde Pública. 2005;39(5):795-801.

6. Moura ERF, Silva RM, Galvão MTG. Dinâmica do atendimento em planejamento familiar no Programa Saúde da Família no Brasil. Cad Saúde Pública. 2007;23(4):961-970.

7. Oguisso T, Freitas GF. Enfermeiros prescrevendo medicamentos: possibilidades e perspectivas. Rev Bras Enferm. 2007;60(2):141-144.

8. Ximenes Neto FRG, Costa FAM, Chagas MIO, Cunha ICKO. Olhares dos enfermeiros acerca de seu processo de trabalho na prescrição medicamentos na Estratégia Saúde da Família. Rev Bras Enferm. 2007;60(2):133-140.

9. Buchan J, Calman L. Implementing nurse prescribing: updated review of current practice internationally. Geneva: ICN; 2004

10. Pierre LAS, Clapis MJ. Planejamento familiar em Unidade de Saúde da Família. Rev Latino-Am Enferm. 2010;18(6):8.
11. Parreira BDM, Silva SR, Miranzi MAS. Métodos anticoncepcionais: orientações recebidas por puérperas no pré-natal e puerpério. Ciênc Cuid Saúde. 2010;9(2):262-268.

12. Costa MM, Crispim ZM. Política de saúde do planejamento familiar na ótica do enfermeiro. Rev Enferm UFPE on line. 2010;4(2):568-76.

13. Ministério da Saúde. Secretaria de Políticas de Saúde. Assistência em planejamento familiar: manual técnico. Brasília, DF: Ministério da Saúde; 2002.

14. Andrade EC, Silva LR. Planejamento familiar: uma questão de escolha. Rev Eletrônica Enferm. [periódico na internet]. 2009 [acesso em 13 mar 2011];11(1):85-93. Disponível em: http://www.fen.ufg.br/revista/v11/n1/v11n1a11.htm.

15. Osis MJD, Duarte GA, Crespo ER, Espejo X, Pádua KS. Escolha de métodos contraceptivos entre usuárias de um serviço público de saúde. Cad Saúde Pública. 2004;20(6):1586-94.

16. Carvalho MLO, Schor N. Motivos de rejeição aos métodos contraceptivos reversíveis em mulheres esterilizadas. Rev Saúde Pública. 2005;39(5):788-794.

17. Osis MJD, Faúndes A, Makuch MY, Mello MB, Sousa MH Araújo MJO. Atenção ao planejamento familiar no Brasil hoje: reflexões sobre os resultados de uma pesquisa. Cad Saúde Pública. 2006;22(11): 2481-2490.

18. Moura ERF, Silva RM. Informação e planejamento familiar como medidas de promoção da saúde. Ciênc Saúde Coletiva. 2004;9(4):1023-1032.

19. Balbino EE, Dias MF. Farmacovigilância: um passo em direção ao uso racional de plantas medicinais e fitoterápicos. Rev Bras Farmacogn. 2010;20(6):992-1000.

20. Urwin J. Contraception. Choice advice. Nurses Times [periódico na internet]. 1994 [acesso em 03 mar 2011];90(26):56-58. Disponível em: http://www.ncbi. nlm.nih.gov/pubmed/8047443. 